\title{
Nonstationary Westward Translation of Nonlinear Frontal Warm-Core Eddies
}

\author{
ANGELO RUBinO \\ Dipartimento di Scienze Ambientali, Università Ca'Foscari, Venice, Italy \\ SERGEY DOTSENKo \\ Marine Hydrophysical Institute, Sevastopol, Ukraine \\ PETER BRANDT \\ IFM-GEOMAR, Leibniz-Institut für Meereswissenschaften, Kiel, Germany
}

(Manuscript received 1 July 2008, in final form 18 November 2008)

\begin{abstract}
For the first time, an analytical theory and a very high-resolution, frontal numerical model, both based on the unsteady, nonlinear, reduced-gravity shallow water equations on a $\beta$ plane, have been used to investigate aspects of the migration of homogeneous surface, frontal warm-core eddies on a $\beta$ plane. Under the assumption that, initially, such vortices are surface circular anticyclones of paraboloidal shape and having both radial and azimuthal velocities that are linearly dependent on the radial coordinate (i.e., circular pulsons of the first order), approximate analytical expressions are found that describe the nonstationary trajectories of their centers of mass for an initial stage as well as for a mature stage of their westward migration. In particular, near-inertial oscillations are evident in the initial migration stage, whose amplitude linearly increases with time, as a result of the unbalanced vortex initial state on a $\beta$ plane. Such an initial amplification of the vortex oscillations is actually found in the first stage of the evolution of warm-core frontal eddies simulated numerically by means of a frontal numerical model initialized using the shape and velocity fields of circular pulsons of the first order. In the numerical simulations, this stage is followed by an adjusted, complex nonstationary state characterized by a noticeable asymmetry in the meridional component of the vortex's horizontal pressure gradient, which develops to compensate for the variations of the Coriolis parameter with latitude. Accordingly, the location of the simulated vortex's maximum depth is always found poleward of the location of the simulated vortex's center of mass. Moreover, during the adjusted stage, near-inertial oscillations emerge that largely deviate from the exactly inertial ones characterizing analytical circular pulsons: a superinertial and a subinertial oscillation in fact appear, and their frequency difference is found to be an increasing function of latitude. A comparison between vortex westward drifts simulated numerically at different latitudes for different vortex radii and pulsation strengths and the corresponding drifts obtained using existing formulas shows that, initially, the simulated vortex drifts correspond to the fastest predicted ones in many realistic cases. As time elapses, however, the development of a $\beta$-adjusted vortex structure, together with the effects of numerical dissipation, tend to slow down the simulated vortex drift.
\end{abstract}

\section{Introduction}

Much work has been devoted during the past few decades toward the understanding of the $\beta$-induced movement of geophysical oceanic and atmospheric vortices (see, e.g., Warren 1967; Firing and Beardsley 1976; Flierl 1977; Nof 1981; Killworth 1983; Nof 1983;

Corresponding author address: Angelo Rubino, Dipartimento di Scienze Ambientali, Università Ca' Foscari di Venezia, Calle Larga Santa Marta, Dorsoduro 2137, I-30123 Venezia, Italy.

E-mail: rubino@unive.it
Larichev 1984; Dewar 1988; Cushman-Roisin et al. 1990; Reznik and Dewar 1994; Benilov 1996; Nycander 2001; Graef 1998; Ripa 1997; McDonald 1998; Reznik and and Grimshaw 2001; van Leeuwen 2007). In the ocean, on a $\beta$ plane, steady frontal surface anticyclones of the reduced-gravity equations experience a westward translation in order to compensate for the net Coriolis force on the vortex's rotation velocity field (see, e.g., Flierl et al. 1983; Dewar 1988). The resulting migration can be described, essentially, as a westward shift in conjunction with inertial oscillations of the vortex's center of mass (see, e.g., Nof 1984; van Leeuwen 2007). 
Apart from the limitations inherent in the reducedgravity equations (they do not allow, e.g., for energy decay due to baroclinic instabilities or internal waves radiation), the vortices considered in the past when obtaining such translation formulas are characterized by a simplified dynamics, as their swirl velocities were assumed to be geostrophic and/or steady (see, e.g., Nof 1984; van Leeuwen 2007). However, analytical solutions describing the dynamics of nonlinear, surface frontal anticyclones of the reduced-gravity equations on an $f$ plane indicate that the vortex velocity fields may deviate considerably from the geostrophic equilibrium and that the vortex's shape and velocity structure are intrinsically unsteady. These vortices, in fact, are characterized by inertial oscillations affecting both the radial and tangential velocities and, hence, the vortex's interfacial depth: they deepen (contract) and shallow (expand) during an exact inertial period (Cushman-Roisin 1986; Rubino et al. 1998). In these analytical solutions ("pulson" solutions), the vortex's radial velocity field is a linear function of the vortex's radius, while the azimuthal velocity field can exhibit a complex shape and can deviate considerably from geostrophy (Rubino et al. 1998; Dotsenko and Rubino 2006). Note that the dynamical characteristics mentioned above have been proven 1) to be robust features of frontal surface homogeneous anticyclones simulated numerically and produced experimentally (see Rubino et al. 2002; Rubino and Brandt 2003) and 2) to extend also to theoretical stratified surfaces as well as intermediate lenses (Rubino and Dotsenko 2006; Dotsenko and Rubino 2006).

It can be thus conjectured that a similar, intrinsically unsteady, noticeably ageostrophic behavior may also characterize frontal surface anticyclones evolving on a $\beta$ plane. If this is true, it is conceivable that dynamics similar to those characterizing pulsonlike vortices will also occur on a $\beta$ plane.

In the present paper, we use an analytical theory and a very high-resolution, frontal numerical model, both based on the unsteady nonlinear, reduced-gravity shallow water equations on a $\beta$ plane, to explore aspects of the migration of homogeneous surface, frontal warmcore eddies on a $\beta$ plane. The paper is organized as follows: in section 2 the adopted models are described; in section 3 aspects of the nonlinear, unsteady vortex dynamics obtained analytically and simulated numerically on a $\beta$ plane are discussed; and finally, in section 4 , the results are discussed and conclusions presented.

\section{Analytical and the numerical model}

Let us consider a homogeneous, circular surface buoyant layer of density $\rho_{1}$ that, at the sea surface, is

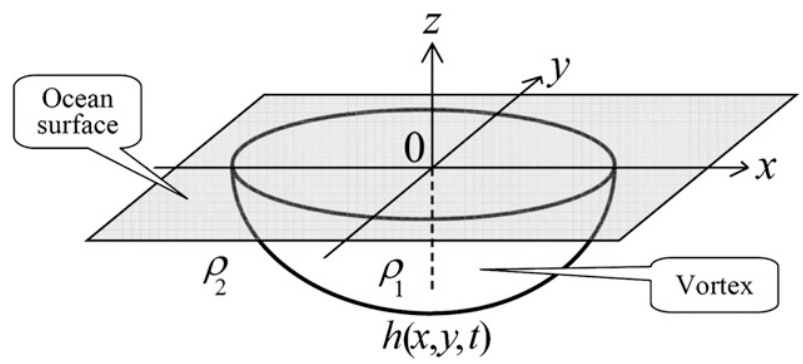

FIG. 1. Initial shape of the surface vortex on a $\beta$ plane (circular pulson of the first order). Here, $h$ is the vortex thickness, and $\rho_{1}$ and $\rho_{2}$ are the vortex and the ambient density, respectively.

separated from the surrounding, infinitely deep, quiescent ocean having density $\rho_{2}>\rho_{1}$ by a closed frontal line (Fig. 1). Within the framework of the nonlinear, unsteady, reduced-gravity shallow-water equations on a $\beta$ plane, the dynamics of such a water anomaly is governed by the following system of equations:

$$
\begin{aligned}
& \frac{\partial u}{\partial t}+u \frac{\partial u}{\partial x}+v \frac{\partial u}{\partial y}-f v=-g^{\prime} \frac{\partial h}{\partial x}, \\
& \frac{\partial v}{\partial t}+u \frac{\partial v}{\partial x}+v \frac{\partial v}{\partial y}+f u=-g^{\prime} \frac{\partial h}{\partial y}, \quad \text { and } \\
& \frac{\partial h}{\partial t}+\frac{\partial(h u)}{\partial x}+\frac{\partial(h v)}{\partial y}=0,
\end{aligned}
$$

where $x, y$ are the zonal and the meridional spatial coordinates, respectively; $t$ is the time; $u, v$ the vertically averaged zonal and meridional components, respectively, of the horizontal velocity; $h$ the vortex thickness; $f=f_{0}+\beta y$ the Coriolis parameter; and $g^{\prime}=g\left(1-\rho_{1} / \rho_{2}\right)$ the reduced gravity.

We impose the following initial conditions on Eqs. (1)-(3):

$$
u=u_{0}(x, y), \quad v=v_{0}(x, y), \quad h=h_{0}(x, y), \quad t=0 .
$$

Let us now choose the initial fields (4) so that they represent a pulson of the first order (Cushman-Roisin 1987; Rubino et al. 1998), that is, a circular frontal anticyclone of paraboloidal shape with radial as well as azimuthal velocities that are both linear functions of the vortex radius:

$$
\begin{aligned}
& u=\frac{1}{2} \gamma f_{0} \cos \Phi \Psi x+\left(\frac{1}{2} f_{0}-l \Psi\right) y, \\
& v=-\left(\frac{1}{2} f_{0}-l \Psi\right) x+\frac{1}{2} \gamma f_{0} \cos \Phi \Psi y, \text { and } \\
& h=H \Psi\left(1-\Psi \frac{x^{2}+y^{2}}{W^{2}}\right),
\end{aligned}
$$


where

$$
\Psi=\frac{1}{1+\gamma \sin \Phi}, \quad \Phi=f_{0} t+\phi
$$

In the previous expressions, $H$ and $W$ are, respectively, the maximum vortex thickness and the vortex radius for $\Phi=0$. Note that the parameter $\gamma$ determines the vortex's oscillation strength, while $\varphi$ is the initial phase of the vortex's oscillations, and

$$
l=\frac{1}{2} f_{0} \sqrt{1-\gamma^{2}-8 \mathrm{Ro}^{2}}, \quad \text { Ro }=\frac{\sqrt{g^{\prime} H}}{f_{0} W},
$$

where Ro is the vortex's Rossby number.

Note that the expressions (5)-(8) are the known, exact pulson solutions as obtained for an $f$ plane by Cushman-Roisin (1987). They are not valid on a $\beta$ plane. For further details about the pulson solution, the reader is referred to the excellent paper by CushmanRoisin (1987).

For simplicity we set in the present investigation $\varphi=$ $-\pi / 2$. Note that, at the sea surface, the vortex is bounded by the movable outcropping circular line with oscillating radius described by $x^{2}+y^{2}=W^{2} / \Psi$. Because of (9), moreover, the following inequality must be satisfied:

$$
|\gamma| \leq \Gamma, \quad \Gamma=\sqrt{1-8 \mathrm{Ro}^{2}} .
$$

The vortex's volume $(Q)$, potential $(P)$, and kinetic $(K)$ energy are

$$
\begin{aligned}
Q & =\int h d S, \\
P & =\frac{1}{2} \rho_{1} g^{\prime} \int h^{2} d S, \text { and } \\
K & =\frac{1}{2} \rho_{1} \int h\left(u^{2}+v^{2}\right) d S,
\end{aligned}
$$

where the integrals extend to the area of nonnegative vortex thickness. The total energy of the vortex, which is conserved, as no energy loss can occur in the inviscid reduced gravity model, is thus $E=K+P$. In the case of the pulson of the first order described by (5)-(7), we have

$$
\begin{aligned}
Q & =\frac{1}{2} \pi H W^{2}, \\
P & =\frac{1}{6} \pi \rho_{1} g^{\prime} H^{2} W^{2} \Psi, \quad K=E-P, \quad \text { and } \\
E & =\frac{1}{24} \pi\left(1-\sqrt{\Gamma^{2}-\gamma^{2}}\right) \rho_{1} f_{0}^{2} H W^{4} .
\end{aligned}
$$
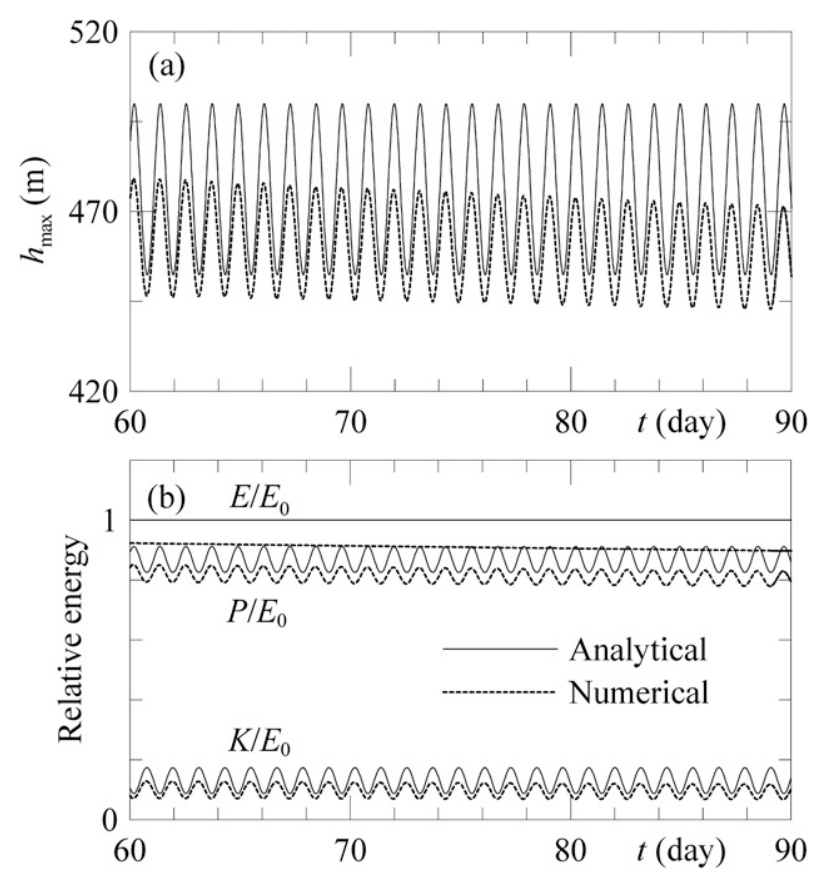

FIG. 2. Comparison between the analytical solution and a numerical simulation referring to a circular pulson of the first order on an $f$ plane with $\rho_{1}=10^{3} \mathrm{~kg} \mathrm{~m}^{-3}, g^{\prime}=0.01 \mathrm{~m} \mathrm{~s}^{-2}, H=500 \mathrm{~m}$, $W=150 \mathrm{~km}, \gamma=0.05$, and $\phi_{0}=25^{\circ}$. (a) The temporal evolution of analytical and numerical vortex thicknesses, and (b) the temporal evolution of analytical and numerical normalized vortex total, potential and kinetic energies.

The previous formula indicates that the total vortex energy is a function also of the vortex's oscillation strength $\gamma$ [see also Eqs. (5)-(7)]. Thus, given a vortex having a certain average shape, its total energy is a function of $\gamma$ only.

Equations (1)-(3) have been solved numerically using a frontal numerical model similar to that presented by Rubino et al. (2002) and Rubino and Brandt (2003), to whose works the reader is referred for further details on the implemented numerical techniques. In this model, based on a staggered Arakawa C grid of $1200 \times 1200$ nodes having a grid spacing of $500 \mathrm{~m}$, a special technique for the treatment of movable lateral boundaries is implemented, which allows for vortex expansions, contractions, and translations. On an $f$ plane, the model is able to reproduce accurately the nonlinear, unsteady pulson dynamics. In Fig. 2, a comparison between pulson characteristics obtained analytically and simulated numerically is presented. After 90 inertial periods, the simulated vortex is still characterized by strong inertial pulsations, while only about $10 \%$ of its initial total energy is numerically dissipated, mostly as a result of unavoidable inaccuracies emerging especially at the vortex's frontal line. 
To explore aspects of the nonlinear, unsteady pulson dynamics on a $\beta$ plane, we first used, as the initial conditions, the pulson solution with the following parameters: $\rho_{1}=10^{3} \mathrm{~kg} \mathrm{~m}^{-3} ; g^{\prime}=0.01 \mathrm{~m} \mathrm{~s}^{-2} ; W=150 \mathrm{~km}$; central latitude $\phi_{0}=20^{\circ}, 25^{\circ}, 30^{\circ}, 35^{\circ}$, and $40^{\circ}$; and $-0.1 \leq \gamma \leq 0.1$. Note that in our simulations the initial thickness of the vortex $H /(1-\gamma)$ was defined to be $500 \mathrm{~m}$. Given the selected vortex radius $W$, the whole vortex diameter is resolved in 600 grid cells. The chosen initial conditions do not represent an exact solution of the model equations. Consequently, we expect an adjustment phase to take place that is not dissimilar (but weaker) from those induced, for example, by the impulsive vortex release investigated by Csanady (1979) and Rubino and Brandt (2003).

\section{Vortex dynamics on a $\beta$ plane}

\section{a. Motion of the center of mass}

The coordinates of the vortex's center of mass $(X, Y)$ are defined as

$$
X=\frac{1}{Q} \int x h d S, \quad Y=\frac{1}{Q} \int y h d S .
$$

The velocity translation of the vortex's center of mass can be derived by a temporal differentiation of (17) using Eqs. (1)-(3) (see Ball 1963; Cushman-Roisin 1986):

$$
\frac{d X}{d t}=\frac{1}{Q} \int h u d S, \quad \frac{d Y}{d t}=\frac{1}{Q} \int h v d S .
$$

A temporal differentiation of (18) leads to the following system of equations:

$$
\frac{d^{2} X}{d t^{2}}-f_{0} \frac{d Y}{d t}=F_{1}, \quad \frac{d^{2} Y}{d t^{2}}+f_{0} \frac{d X}{d t}=F_{2}
$$

where

$$
F_{1}=\frac{\beta}{Q} \int y h v d S, \quad F_{2}=-\frac{\beta}{Q} \int y h u d S .
$$

If we suppose that the vortex's velocity field should be approximately in geostrophic equilibrium, that is,

$$
u \approx-\frac{g^{\prime}}{f} \frac{\partial h}{\partial y}, \quad v \approx \frac{g^{\prime}}{f} \frac{\partial h}{\partial x},
$$

then the integrals (20) may be written as

$$
F_{1}=\frac{\beta g^{\prime}}{2 Q} \int \frac{y}{f} \frac{\partial h^{2}}{\partial x} d S=0 \quad \text { and }
$$

$$
\begin{aligned}
F_{2} & =\frac{\beta g^{\prime}}{2 Q} \int \frac{y}{f} \frac{\partial h^{2}}{\partial y} d S=-\frac{\beta g^{\prime}}{2 Q} \int \frac{\partial}{\partial y}\left(\frac{y}{f}\right) h^{2} d S \\
& \approx-\frac{\beta g^{\prime}}{2 Q f_{0}} \int h^{2} d S .
\end{aligned}
$$

Let us assume now that the vortex's deformations do not essentially modify the quantity $\int h^{2} d S$, which is proportional to the vortex's potential energy [see Eq. (15)]. Note that, within the framework of frontal geostrophy, such a quantity is exactly conserved (see CushmanRoisin 1986). With such an assumption, we can consider

$$
F_{2}=f_{0} C_{\beta}=\text { const }, \quad C_{\beta}=-\frac{\beta g^{\prime}}{2 Q f_{0}^{2}} \int h_{0}^{2} d S<0,
$$

where $C_{\beta}$ is the vortex's westward drift. In this case, the westward drift of the vortex described by (5)-(7) is

$$
C_{\beta}=-\frac{\beta g^{\prime} H}{3 f_{0}^{2}},
$$

which corresponds, essentially, to the formulas found by Nof (1981) and Cushman-Roisin (1986).

The system in (19) can be solved analytically. Due to the axial symmetry of the vortex at $t=0$, the initial conditions for (19) are [see also (18)]

$$
X(0)=Y(0)=d X(0) / d t=d Y(0) / d t=0 ;
$$

that is, the initial vortex drift vanishes. The general solution of (19), using the assumptions from (21), (23), and (24) and the initial conditions in (26), corresponds to the cycloid

$$
X=\frac{C_{\beta}}{f_{0}}\left(f_{0} t-\sin f_{0} t\right), \quad Y=\frac{C_{\beta}}{f_{0}}\left(1-\cos f_{0} t\right) .
$$

This solution for the trajectory of the vortex's center of mass was recently found by van Leeuwen (2007), while Nof (1984) showed that such a trajectory can be followed by an anomaly of dense water (deep cold eddy) propagating along a sloping bottom.

Supposing that the vortex's paraboloidal shape and linear velocity field remain substantially preserved on a $\beta$ plane, we can find another approximation for the trajectory of the center of mass of the vortex evolving on a $\beta$ plane. Substituting the analytical solution (5)-(7) into (20), in fact, leads to

$$
\begin{aligned}
F_{1} & =a \cos \Phi, \quad F_{2}=-a \sin \Phi-b \quad \text { and } \\
a & =\frac{1}{12} \beta \gamma f_{0} W^{2}, \quad b=\frac{1}{12} \beta f_{0} W^{2}\left(1-\sqrt{\Gamma^{2}-\gamma^{2}}\right) .
\end{aligned}
$$




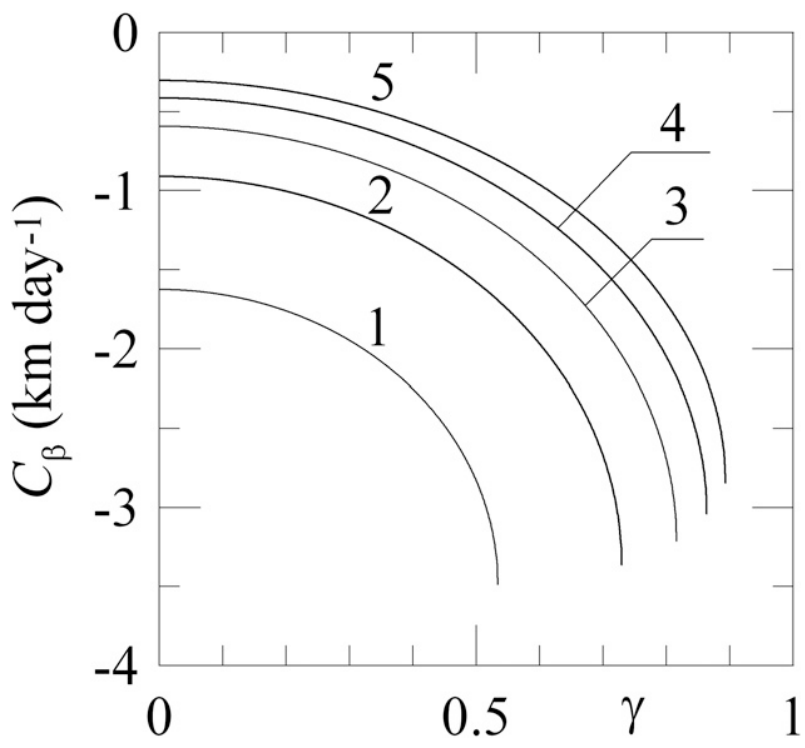

FIG. 3. Dependence of the vortex's westward drift expressed by (30) on vortex amplitude parameter $\gamma\left[0 \leq \gamma \leq \Gamma\left(\phi_{0}\right)\right]$ and on central latitude $\phi_{0}$ : line $1, \phi_{0}=20^{\circ}$; line $2, \phi_{0}=25^{\circ}$; line $3, \phi_{0}=30^{\circ}$; line $4, \phi_{0}=35^{\circ}$; and line $5, \phi_{0}=40^{\circ}$.

In such a case, the solution of (19), with the initial conditions from (26), can be written as

$$
\begin{aligned}
& X=-\frac{a}{f_{0}^{2}}\left(1-\cos f_{0} t-f_{0} t \sin f_{0} t\right)-\frac{b}{f_{0}^{2}}\left(f_{0} t-\sin f_{0} t\right), \\
& Y=\frac{a}{f_{0}^{2}}\left(f_{0} t \cos f_{0} t-\sin f_{0} t\right)-\frac{b}{f_{0}^{2}}\left(1-\cos f_{0} t\right) .
\end{aligned}
$$

The previous formulas in (27) and (29) show that, on a $\beta$ plane, the oscillations of the center of mass of an initially circular pulson of the first order resonantly increase. Also, from the previous formulas, a nonoscillatory vortex's westward drift can be identified:

$$
C_{\beta}=\frac{b}{f_{0}}=-\frac{1}{12} \beta W^{2}\left(1-\sqrt{\Gamma^{2}-\gamma^{2}}\right) .
$$

According to (30), the vortex's westward drift increases for increasing oscillation amplitudes $\gamma$ and central latitudes $\phi_{0}$ (Fig. 3). For $\gamma=0$, (30) takes the form

$$
C_{\beta}=-\frac{1}{12} \beta W^{2}(1-\Gamma),
$$

which corresponds to the westward drift of nonlinear steady vortices of paraboloidal shape and linear velocity fields, as found by Nof (1981). If the vortex's Rossby number is small, the approximation of (31) obtained using the Maclaurin series expansion of (10) to the first

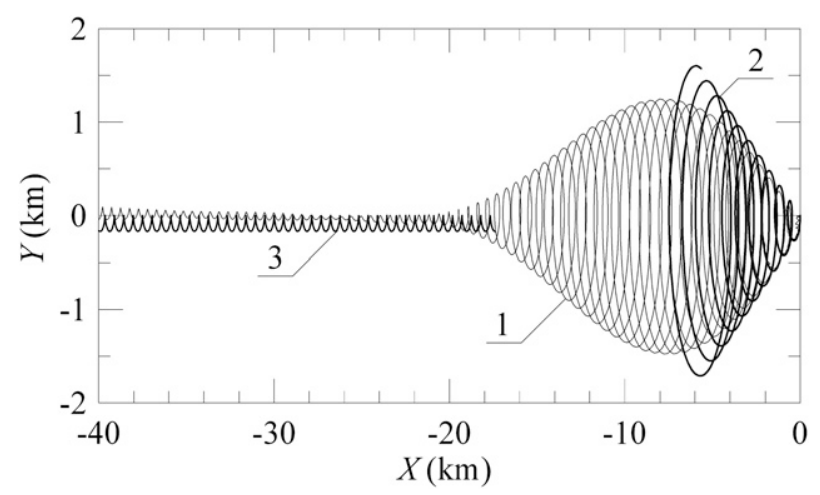

FIG. 4. Trajectory of the vortex center of mass as simulated numerically (line 1) and as obtained analytically using Eq. (29), line 2, and using Eq. (27), line 3. The trajectory depicted by line 2 is obtained by inserting into Eqs. (19) and (20), expressing the evolution of the vortex center of mass on a $\beta$ plane, the exact structure of a pulson ( $f$-plane solution) as in the initial condition. The trajectory depicted by line 3 is obtained assuming geostrophic velocities in Eqs. (19) and (20). We refer to the situation depicted by line 2 as being the "initial" stage and to that depicted by line 3 as being the "mature" stage.

order coincides with (25). Thus, oscillating vortices migrate faster than the corresponding nonoscillating ones, and nonlinear vortices migrate faster than linear ones. The expressions (29) and (30) address, for the first time, the evolution of an oscillating eddy initially not adjusted to the unsteady nonlinear $\beta$-plane equations.

Note that the expressions (29) and (27) can be considered to be referring to the initial stage and to a mature stage of the development of a circular pulson of the first order evolving on a $\beta$ plane. In Fig. 4 we present a comparison between the analytical expressions (29) and (27) and their counterparts obtained numerically by solving the complete set of the unsteady, nonlinear reduced-gravity shallow-water equations. The first stage of the development of an initially circular pulson on a $\beta$ plane simulated numerically is, indeed, characterized by an increase of the inertial oscillations marking the route of its center of mass (Fig. 4a), which is derived from the unbalanced vortex's initial state on a $\beta$ plane. As time elapses, however, the simulated oscillation amplitude decreases, and the simulated trajectory closely resembles that obtained analytically under the assumption of the existence of an almost geostrophic equilibrium (Fig. 4b).

\section{b. Frequency structure and westward drift}

The modification of the spatial structure of an initially circular pulson induced by the presence of the $\beta$ effect is evidenced in Fig. 5, where the numerical evolution of its maximum thickness and of its center of mass are delineated. Due to the $\beta$ effect, an asymmetry in the 

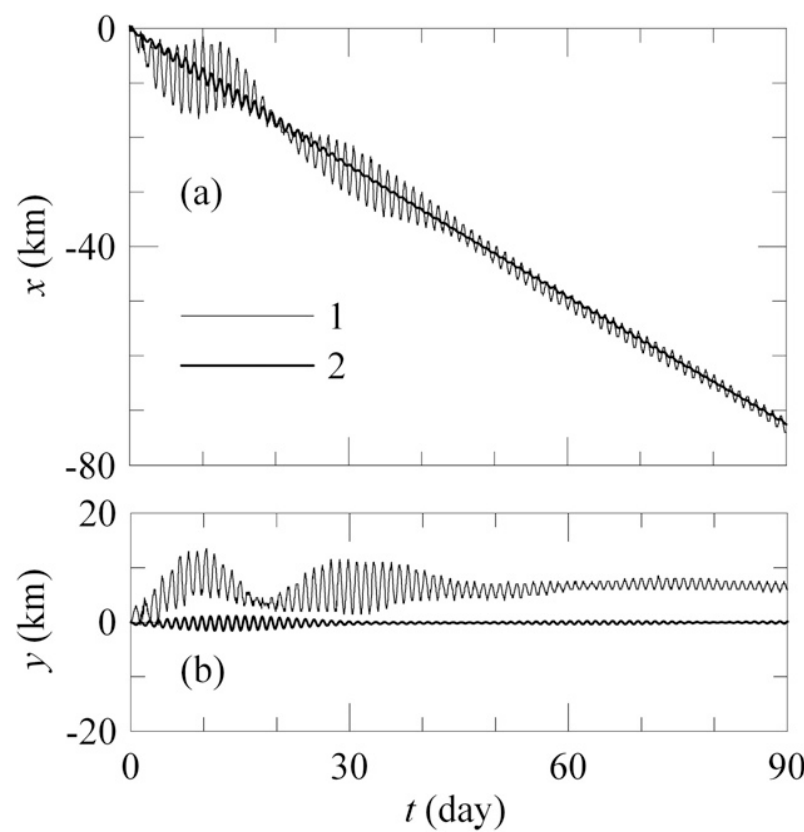

FIG. 5. Temporal evolution of the (a) zonal $x$ and (b) meridional $y$ coordinates of the maximum vortex thickness (line 1) and of the vortex center of mass (line 2) for $\gamma=0.05$ and $\phi_{0}=25^{\circ}$ as obtained numerically.

meridional component of the vortex horizontal pressure gradient develops to compensate for the variation of the Coriolis parameter with latitude: The vortex shape will be steeper on its poleward side than on its equatorward side. Accordingly, the location of the simulated vortex maximum depth is always found poleward of the simulated vortex's center of mass (Fig. 5b).

Most noticeably, even the intrinsic temporal oscillatory structure characterizing the circular pulson on an $f$-plane experiences a profound transformation when the vortex evolves on a $\beta$ plane. In Fig. 6 the evolution of the maximum thickness of an initially circular pulson evolving on a $\beta$ plane is depicted for five different central latitudes, $f_{0}$. In all the simulated cases, the exactly inertial, monochromatic pulsation mode, characteristic of the circular pulson dynamics, splits into a bimodal structure, made of a superinertial and a subinertial pulsation. Accordingly, a typical beat structure develops, with amplifications and attenuations modulated by the frequency difference between the two modes, which is found to be a monotonically decreasing function of latitude (Fig. 7).

A comparison between the westward propagation described by the formulas of Nof (1981) and CushmanRoisin (1986) and that obtained numerically using our frontal model is depicted in Fig. 8 for five different central latitudes, $f_{0}$. In general, the simulated translation
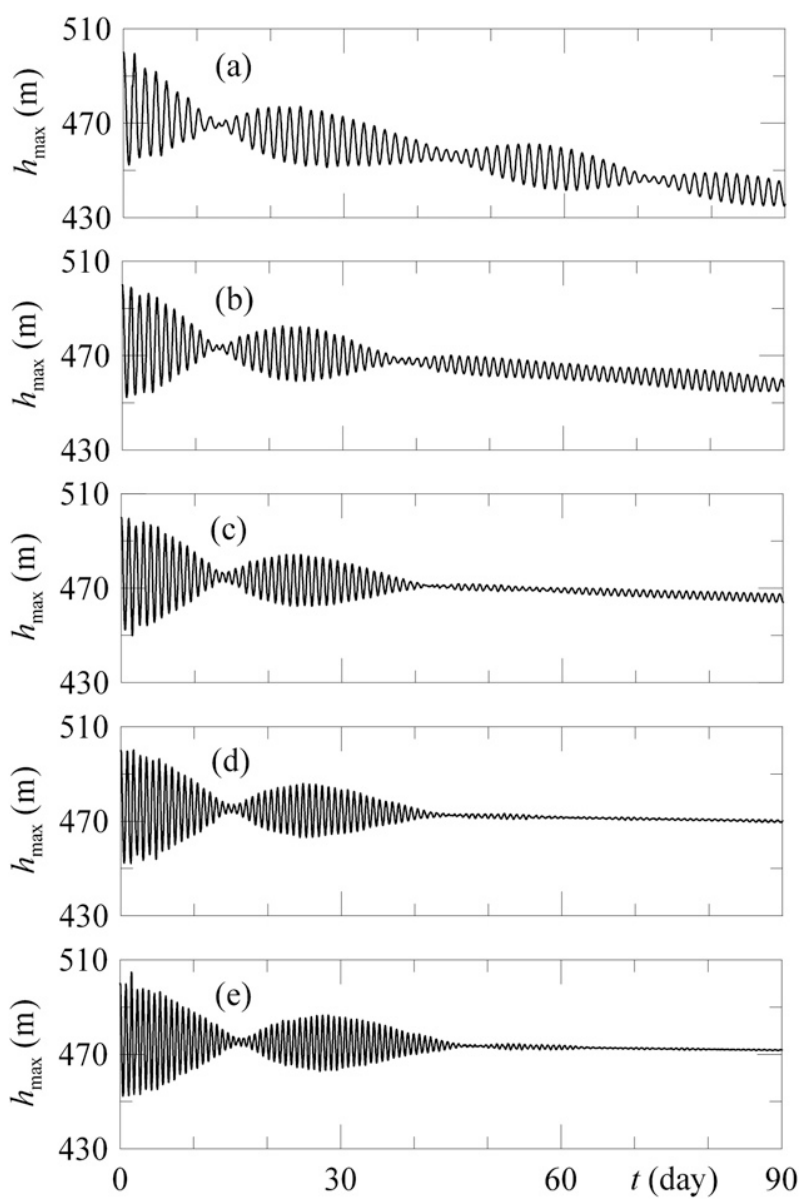

FIG. 6. Temporal evolution of the maximum vortex thickness for different latitudes: $\phi_{0}=$ (a) $20^{\circ}$, (b) $25^{\circ}$, (c) $30^{\circ}$, (d) $35^{\circ}$, and (e) $40^{\circ}$. Note that for all experiments $\gamma=0.05$ and $W=$ $150 \mathrm{~km}$.

is faster than the one predicted by Eq. (25) but it is slower than the one predicted for steady nonlinear vortices, which translate with velocity (31).

The difference between the vortex's westward drift simulated numerically and that obtained analytically (31) decreases with latitude. In particular, for a pulsation strength $\gamma=0.05$, the relative average difference spans from about $3 \%\left(\phi_{0}=40^{\circ}\right)$ to about $10 \%\left(\phi_{0}=\right.$ $20^{\circ}$ ). Note that such differences would be even smaller in the limit of vanishing numerical dissipation (see also Fig. 2).

However, if we focus our comparison on the initial stage of the vortex evolution on a $\beta$ plane (Fig. 8b), we recognize that the analytical nonlinear predictions based on (31) and the obtained numerical results are very similar, the average numerical drift being even larger than the corresponding analytical one during the first 10 days in the case of large central latitude. 


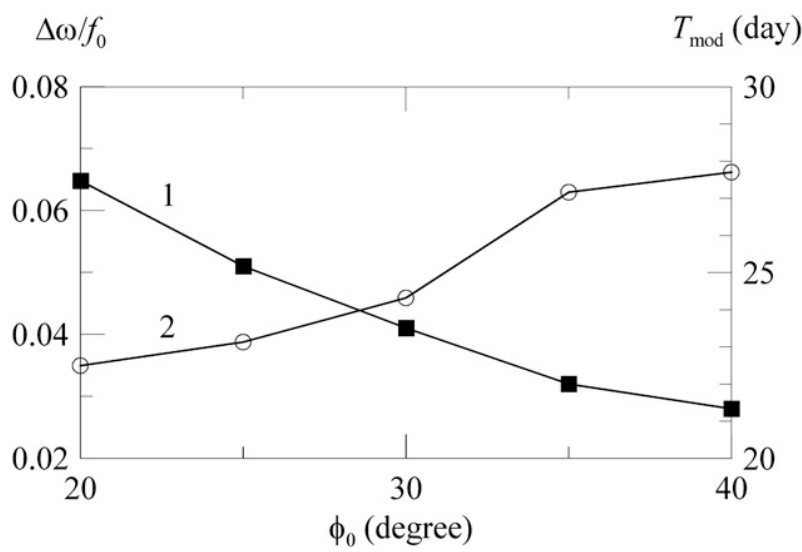

FIG. 7. Normalized frequency difference $\Delta \omega / f_{0}$ between the superinertial and subinertial frequencies of the vortex oscillations (1) and the corresponding periods of modulation, $T_{\bmod }=2 \pi / \Delta \omega(2)$ for $\gamma=0.05$. The frequency difference is obtained by a Fourier analysis of the time series of the maximum vortex thickness.

\section{Discussion and conclusions}

In the present paper, for the first time, aspects of the unsteady, nonlinear dynamics of geophysical frontal vortices of the reduced-gravity shallow-water equations evolving on a $\beta$ plane have been investigated using analytical theories together with the results of a frontal numerical model. We found that an initially circular geophysical frontal vortex having a paraboloidal shape and horizontal velocities that are both linear functions of the spatial coordinates (i.e., a pulson of the first order) is profoundly transformed by the presence of the $\beta$ effect. The exactly inertial pulsations characterizing the pulson dynamics can no longer survive on a $\beta$ plane. They are replaced by a bimodal pulse, namely, a superinertial and a subinertial oscillation leading to a beat, whose period is found to be an increasing function of latitude. Under the assumption that the vortex retains substantially its initial pulsonlike structure, we found analytically that it must develop resonant amplifications of the oscillating position of its center of mass as a result of its initially unbalanced state on a $\beta$ plane. Such analytical initial oscillations encompass a westward drift expression that is a function also of the vortex oscillation strength $\gamma$ [see Eq. (30)]. The frontal numerical model, which solves the complete set of nonlinear, unsteady reduced-gravity shallow-water equations and is prone to different decay processes like, for example, numerical dissipation and filamentation, is able to simulate this initial analytical vortex evolution. Initially, the simulated westward drift agrees very well with that predicted by Nof (1981) for nonlinear vortices [see Eq. (31)]. In the vortex's mature stage of drift, however, the simulated westward drift decreases noticeably, possibly due
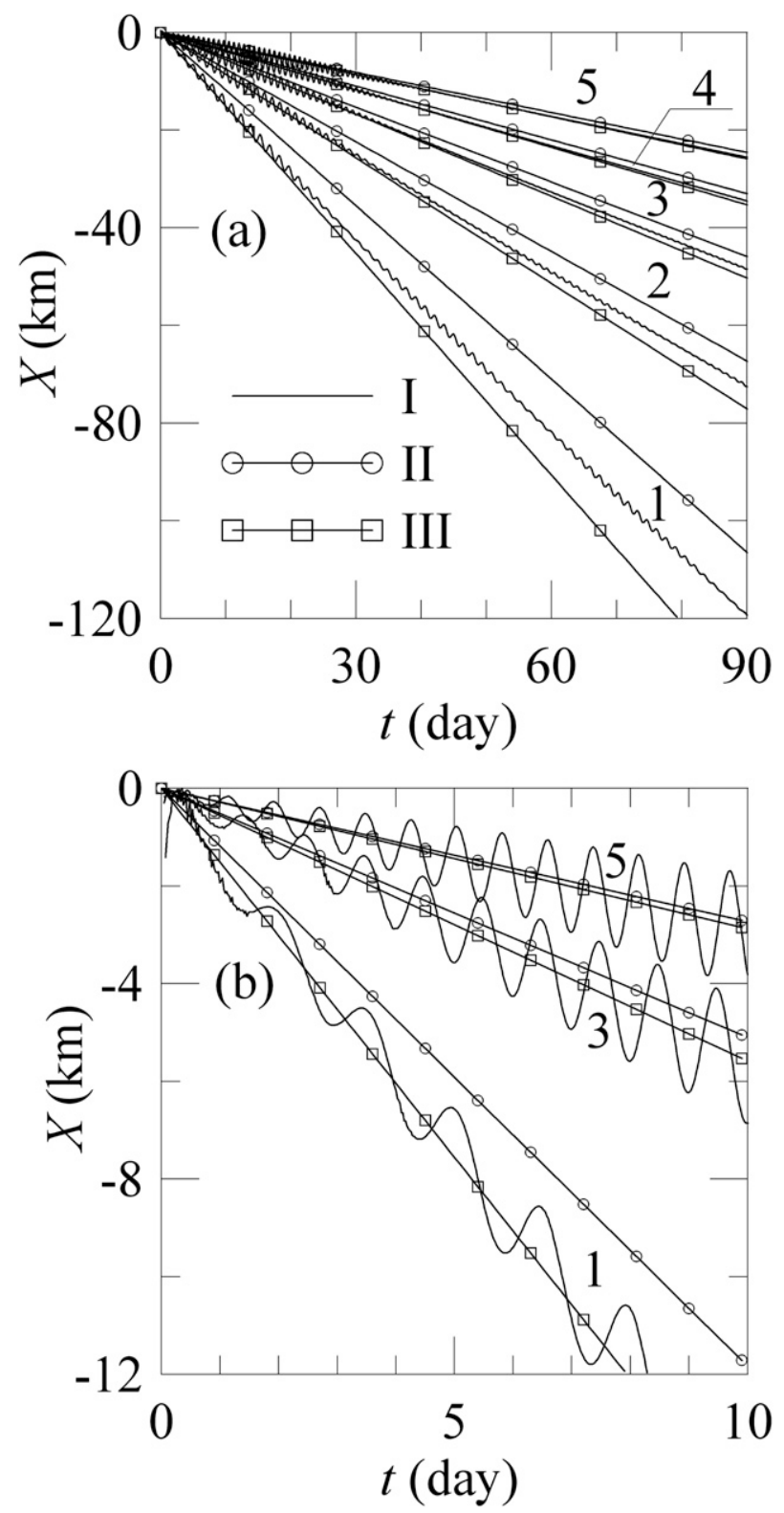

FIG. 8. Comparison between the westward drift of an initially circular pulson of the first order on a $\beta$ plane simulated numerically, line I, and that calculated analytically using (25), line II, and (31), line III, at different central latitudes: $\phi_{0}=20^{\circ}$ (lines 1$), 25^{\circ}$ (lines 2), $30^{\circ}$ (lines 3), $35^{\circ}$ (lines 4), and $40^{\circ}$ (lines 5). (a) The evolution on a 90-day temporal interval, and (b) the evolution of the first 10 days. The calculations were performed using $\gamma=0.05$ and $W=150 \mathrm{~km}$.

to the unavoidable dissipative effects inherent in the complex numerics of the frontal model and to the development of instabilities. The increasing difference obtained between the found analytical solution (29) and the corresponding numerical evolution of the vortex center of mass (see Fig. 4 for small $x$ ) accounts for the 

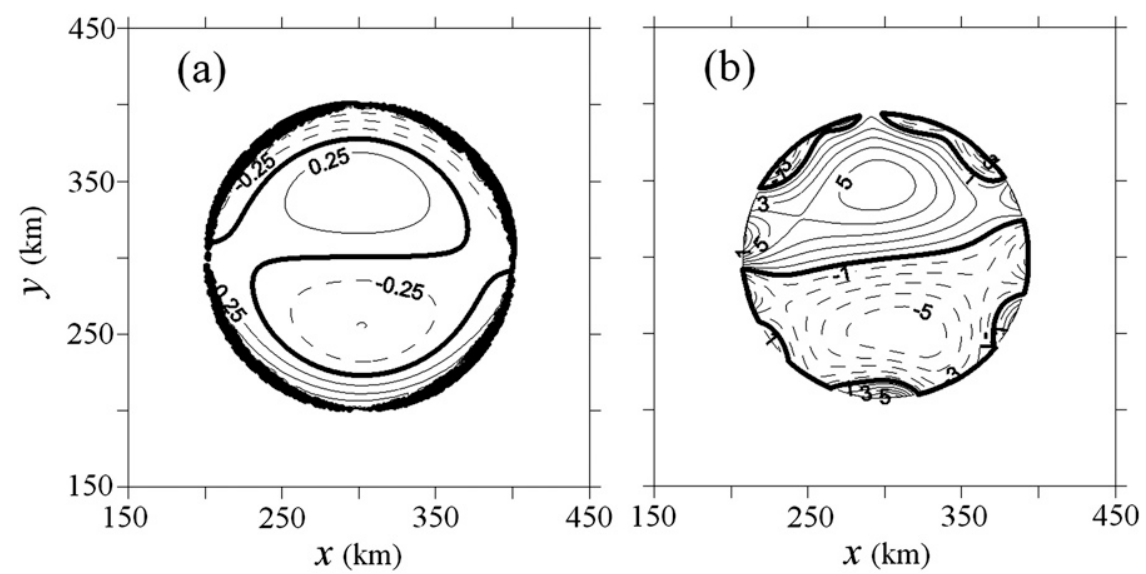

FIG. 9. Difference between the interface position of a vortex evolving on a $\beta$ plane and an initially identical one evolving on an $f$ plane for (a) $t=2 \mathrm{~h}$ and (b) $t=60$ days of simulation time. Such differences have been calculated by removing the vortex drift with respect to its center of mass for the following vortex parameters: $\rho_{1}=10^{3} \mathrm{~kg} \mathrm{~m}^{-3}, g^{\prime}=0.01 \mathrm{~m} \mathrm{~s}^{-2}, H=500 \mathrm{~m}$, $W=150 \mathrm{~km}, \gamma=0$, and $\phi_{0}=40^{\circ}$. Note that the difference is plotted only for regions where the thicknesses of both vortices are larger than $100 \mathrm{~m}$.

vortex adjustment toward an asymmetric structure: the simulated position of its deepest point, for instance, is found to be always located poleward of the simulated position of its center of mass (Fig. 5b), which implies that the vortex is steeper north rather than south of its center. Such an asymmetry can be evinced also by comparing the simulated shapes of a vortex evolving on an $f$ plane with those of an initially identical one evolving on a $\beta$ plane (Fig. 9). The differences between these two shapes, as calculated by removing the vortex drift with respect to its center of mass, show that, in general, the vortex evolving on a $\beta$ plane is deeper than the vortex evolving on the $f$ plane north rather than south of the common center. Such a mass distribution characteristic, which emerges immediately after an initially circular pulson of the first order starts to evolve on a $\beta$ plane (see Fig. 9a), seems to be a very robust feature: it is well recognizable throughout the simulated vortex evolution (Fig. 9b). To this distribution, which has a counterpart in a similar asymmetry in the vortex velocity field, can be ascribed the peculiar dynamical properties of such vortices on a $\beta$ plane.

Acknowledgments. This work was supported by the International Bureau of the German Federal Ministry of Education and Research (BMBF) under Contract UKR 07/001.

\section{REFERENCES}

Ball, F. K., 1963: Some general theorems concerning the finite motion of a shallow rotating liquid lying on a paraboloid. J. Fluid Mech., 17, 240-256.
Benilov, E. S., 1996: Beta-induced translation of strong isolated eddies. J. Phys. Oceanogr., 26, 2223-2229.

Csanady, G. T., 1979: The birth and death of a warm core ring. J. Geophys. Res., 84, 777-780.

Cushman-Roisin, B., 1986: Frontal geostrophic dynamics. J. Phys. Oceanogr., 16, 132-143.

_ 1987: Exact analytical solution for elliptical vortices of the shallow-water equations. Tellus, 39A, 235-244.

_ mesoscale eddies. J. Phys. Oceanogr., 20, 758-768.

Dewar, W. K., 1988: Ventilating beta plane lenses. J. Phys. Oceanogr., 18, 1193-1201.

Dotsenko, S., and A. Rubino, 2006: Analytical solutions for circular stratified eddies of the reduced-gravity shallow-water equations. J. Phys. Oceanogr., 36, 1693-1702.

Firing, E., and R. C. Beardsley, 1976: The behavior of a barotropic eddy on a $\beta$-plane. J. Phys. Oceanogr., 6, 57-65.

Flierl, G. L., 1977: The application of linear quasigeostrophic dynamics to Gulf Stream rings. J. Phys. Oceanogr., 7, 365-379.

— M. E. Stern, and J. A. Whitehead Jr., 1983: The physical significance of modons. Dyn. Atmos. Oceans, 7, 233-263.

Graef, F., 1998: On the westward translation of isolated eddies. J. Phys. Oceanogr., 28, 740-745.

Killworth, P. D., 1983: On motion of isolated lenses on a betaplane. J. Phys. Oceanogr., 13, 368-376.

Larichev, V. D., 1984: Intergral properties of localized eddies on the beta plane. Izv. Atmos. Ocean. Phys., 20, 654-658.

McDonald, N. R., 1998: The time-dependent behaviour of a spinning disc on a rotating planet: A model for geostrophical vortex motion. Geophys. Astrophys. Fluid Dyn., 87, 253-272.

Nycander, J., 2001: Drift velocity of radiating quasigeostrophic vortices. J. Phys. Oceanogr., 31, 2178-2185.

Nof, D., 1981: On the $\beta$-induced movement of isolated baroclinic eddies. J. Phys. Oceanogr., 11, 1662-1672.

_ 1983: The translation of isolated cold eddies on a sloping bottom. Deep-Sea Res., 30, 171-182. 
— 1984: Oscillatory drift of deep cold eddies. Deep-Sea Res., 31, 1395-1414.

Reznik, G. M., and W. K. Dewar, 1994: An analytical theory of distributed axisymmetric barotropic vortices on the $\beta$ plane. J. Fluid Mech., 269, 301-321.

— tense localized vortex on a beta plane. J. Fluid Mech., 443, 351-376.

Ripa, P., 1997: "Inertial" oscillations and the $\beta$-plane approximation(s). J. Phys. Oceanogr., 27, 633-647.

Rubino, A., and P. Brandt, 2003: Warm-core eddies studied by laboratory experiments and numerical modeling. J. Phys. Oceanogr., 33, 431-435.
—, and S. Dotsenko, 2006: The stratified pulson. J. Phys. Oceanogr., 36, 711-719.

— P. Brandt, and K. Hessner, 1998: Analytical solutions for circular eddies of the reduced-gravity, shallow-water equations. J. Phys. Oceanogr., 28, 999-1002.

— K. Hessner, and P. Brandt, 2002: Decay of stable warm-core eddies in a layered frontal model. J. Phys. Oceanogr., 32, 188-201.

van Leeuwen, P. J., 2007: The propagation mechanism of a vortex on the $\beta$ plane. J. Phys. Oceanogr., 37, 2316-2330.

Warren, B. A., 1967: Notes on translatory movement of rings of current with application to Gulf Stream eddies. Deep-Sea Res., 14, 505-524. 